\title{
Cubic Interpolation Pseudo-Particle Navier-Stokes Formulation Method for Solid Particle-Fluid Interaction in Channel Flow with Cavity
}

\author{
Ahmad Sofianuddin A. Sahak ${ }^{1}$, Nor Azwadi Che Sidik ${ }^{1,}$, Siti Nurul Akmal Yusof ${ }^{1}$ \\ 1 Malaysia - Japan International Institute of Technology (MJIT), University Teknologi Malaysia, Jalan Sultan Yahya Petra,54100 Kuala Lumpur, \\ Malaysia
}

\section{ABSTRACT}

Particles dispersion studies have attracted researchers' interest in understanding the physics behind the phenomena. Fluid-particle transport related to cavity flows is relevant to several natural and engineering applications such as cleaning of process equipment. However, there is no detailed discussion on the flow in the cavity channel with the dispersion of the particles and the combination of the effect of different geometric and fluid parameters with the presence of heat. This study is about predicting the behaviour of the solid particle dispersion in the channel with cavity and the interaction with the surrounding fluid with different geometrical, flow and particles parameters. Cubic Interpolation Pseudo-Particle (CIP) method was applied to solve the hyperbolic, advective term of vorticity transport Navier-Stokes equations coupled with energy equation in the two-dimensional, incompressible and laminar flow over the cavity and Eulerian-Lagrangian approach to model the dispersion of the particles inside the cavity. Flow inside cavity with an aspect ratio $(A R)$ range from 0.5 to 4 , and Reynolds number $(R e)$ from 50 to 1000 were investigated. Validation studies were conducted numerically by evaluating the CIP method on the flow in the channel with the cavity. Qualitative comparisons between the numerical and experimental were evaluated and presented.

Keywords:

Particles; CIP method; cavity; channel

Copyright $\odot 2020$ PENERBIT AKADEMIA BARU - All rights reserved

\section{Introduction}

The study of moving bodies in a flow field draws attention to the researchers in recent years due to its application in many fields. Conventionally, an experimental study was used to get real understanding in fluid-particle flow problem [1-4]. However, it is difficult to recreate the same problem in the laboratory for further experimental investigation. Moreover, in the real-life situation, the study of interested particle flow problem may involve many variable parameters, and some of them maybe not related to the scope of research. Therefore, it took a lot of time to control the variables to generate accurate outcomes. Besides, due to the limitation of the measuring devices, it is very challenging to get precise information for all point of interest in the flow itself for further analysis [5]. Tsorng et al., [6] have highlighted several issues and challenges in their experiment on particle tracking in a flow. Firstly, is the difficulty of tracking particle movement for a long time. There

\footnotetext{
* Corresponding author.

E-mail address: azwadi@utm.my (Nor Azwadi Che Sidik)
}

https://doi.org/10.37934/arms.70.1.117 
are no assurances that these particles will present in the specific target area for analysis due to particles movement. This may lead to the difficulties of tracking multiple particles simultaneously due to the limited capabilities of detection devices. The second challenge is precaution needed to the particles used in the experiment. Finite-size of the particles or inertial migration may result in deviation. This problem also has been raised previously by Nobach and Bodenschatz [5].

However, with the advancement of computing powers over the years, computational fluid dynamics (CFD) nowadays has become an essential instrument for researchers to do the preunderstanding and grasp the first picture of the specific related problem, particularly in engineering application before applying to the full-scale testing. The increasing number of researchers utilizing computerize simulation demonstrate the efficiency of using the approach as it can save time and resources. Also, with the implementation of the CFD, researchers may tackle the complex fluid problems that previously seem to be challenging to reach [7-9]. The complexity is even higher when it comes to analyzing every single point of the particle in the multiphase flow problem, where every variable that affects the flow characteristic is considered.

Cubic-interpolated pseudo-particle (CIP) is one of the methods for solving the general hyperbolic equation. The spatial within each grid is interpolated with a cubic polynomial. Therefore, both values of a specific function and its spatial derivative on the grid are predicted in advance. The primary advantage of the CIP method acquired on a compact stencil is low numerical diffusivity and higher stability of the solution procedure without adapting special treatment as in Yabe and Takei [10]. The CIP model of solving multi-dimensional hyperbolic equation in Yabe et al., [11] for example, can be performed efficiently in vector machines.

Furthermore, it can be extended to increase accuracy since it has been divided into advection and non-advection terms. CIP method is arguably one of the numerical methods that can provide a solution with high accuracy and less diffusive compared to other numerical methods. Therefore, by developing a single particle-laden flow algorithm combining the CIP method for fluid phase with the particles-phase may be beneficial to scientists or engineers to obtain a stable and accurate solution for the related problems.

Over the last few years, Takewaki et al., [12]'s less diffusive CIP method has become very common to solve the hyperbolic equation. However, for spatial gradient data, the initial CIP technique using both the point values and its spatial gradients require additional border circumstances [11-14]. Usually, to get the derivation values on the node, it must distinguish the equation with the spatial variable. The procedure is not difficult for the simple case, where the velocity is constant, but it is not easy for complex equations [15]. The study of the convection process using CIP can be found on the study by Ida [16], Che Sidik et al., [17], and Sheldareh et al., [18]. However, a complete search of the literature revealed that there is no previous research which focuses on utilizing a combination of CIP numerical scheme to assess the flow in a channel with cavity with the particle dispersion and the combining effect of different geometrical and fluid parameters with the presence of heat. This is the motivation of the present study to conduct experimental and numerical analysis on the particle behaviour in the cavity of a channel with a heated cavity using the numerical algorithm based on the established CIP methods. In general, the phrase cavity flow refers to flow over the surface cavity or surface cut-outs. Cavity flows relevant to study the flow regime without implementing complicated geometric structure.

This study aims to investigate the behaviour of the solid particles in the dispersion process from the cavity inside the channel at the various combination of geometrical, flow and particles parameters. CIP method was applied to solve the hyperbolic, advective term of vorticity transport Navier-Stokes equations coupled with energy equation in two-dimensional, incompressible, and 
laminar flow over the cavity and Eulerian-Lagrangian approach to model the dispersion of the particle inside the cavity.

\section{Methodology}

\subsection{Experimental Setup}

Figure 1 illustrates an overview of the experimental layout. The experiment has been conducted in a closed-circuit water channel, which is consists of a tank, that act as the reservoir, centrifugal pump, two valves, flow meter, to indicate the flow rate and also the closed water channel, made with $4 \mathrm{~mm}$ thick acrylic with a different cavity aspect ratio that specially designed for this experiment.

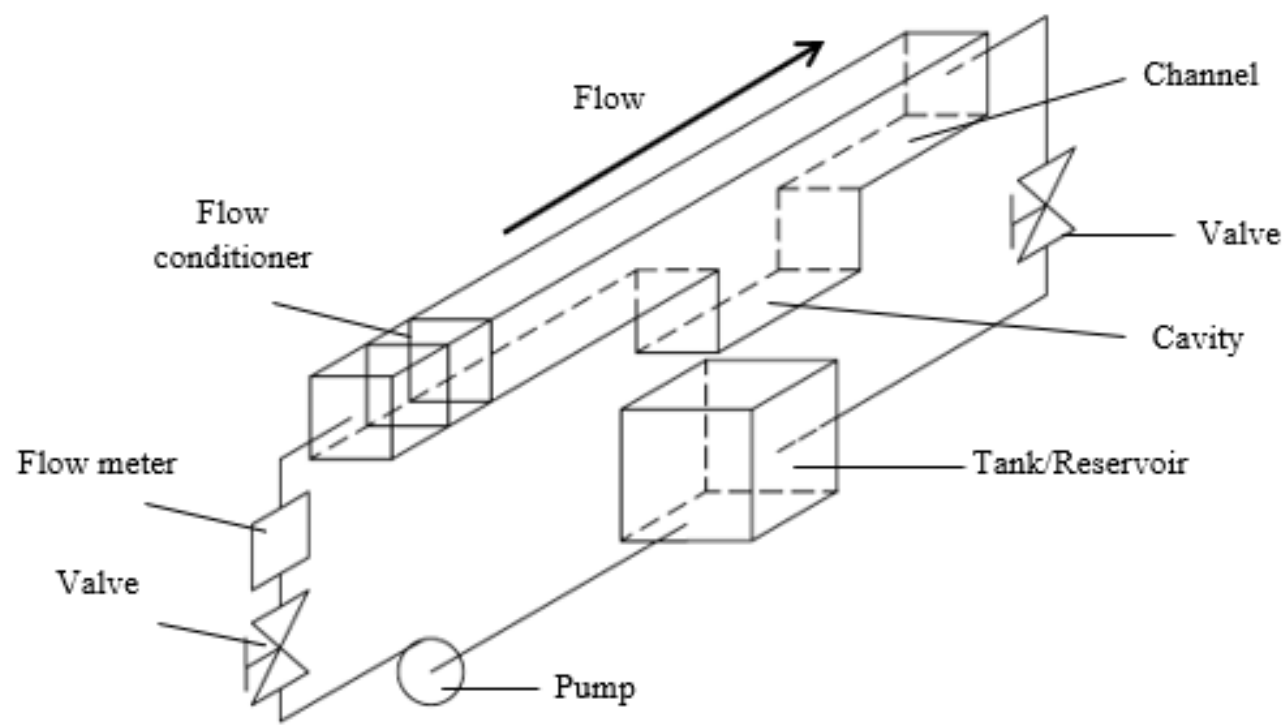

Fig. 1. Diagram of the experimental layout and its component

The entrance length $\left(L_{e}\right)$ is defined as the region where the viscous effects near the wall affect the velocity profile of the flow, which is in constant change [19]. Therefore, it is important to determine the entrance length of the upstream flow so that the velocity profile of the incoming flow becomes constant before reaching the cavity and will not affect the flow formed in the cavity as shown in Figure 2.

The length of the entrance, $L_{e}$ for laminar flow can be written in a function of Reynolds number, $R e$ as follows

$L_{\mathrm{e}}=0.06 R e D_{h}$

where $R e=$ Reynolds number and $D_{h}=$ hydraulic diameter of the pipe. However, because the pipe is not circular, the hydraulic diameter can be described as

$D_{h}=4 A / P$

where $A$ is the area of the cross-section and $P$ is the perimeter of the wet part of the pipe. From Eq. (1), $L_{e}$ for different $R e$ has been calculated and presented in Table 1. 


\section{Table 1}

Length of the entrance, $L_{e}$ required for each of Reynolds number, $R e$

\begin{tabular}{llll}
\hline Reynolds number, $R e$ & 100 & 400 & 1000 \\
Entrance length, $L_{e}(\mathrm{~mm})$ & 150 & 600 & 1500 \\
\hline
\end{tabular}

The test section consists of a smooth $25 \times 25 \mathrm{~mm}$ square channel with $1500 \mathrm{~mm}$ long inlet $\left(L_{i}\right)$, $500 \mathrm{~mm}$ long outlet $\left(L_{o}\right)$ and a wall thickness of $4 \mathrm{~mm}$ for the whole channel and cavity as Figure 3 . For cavity section, the length and depth $(L$ and $H$ ) are the same with the channel opening ( $25 \mathrm{~mm})$ but have a variable range of length, $L$ from 75 to $100 \mathrm{~mm}(A R=3$ and 4). Summary of test section dimension was shown in Table 2.

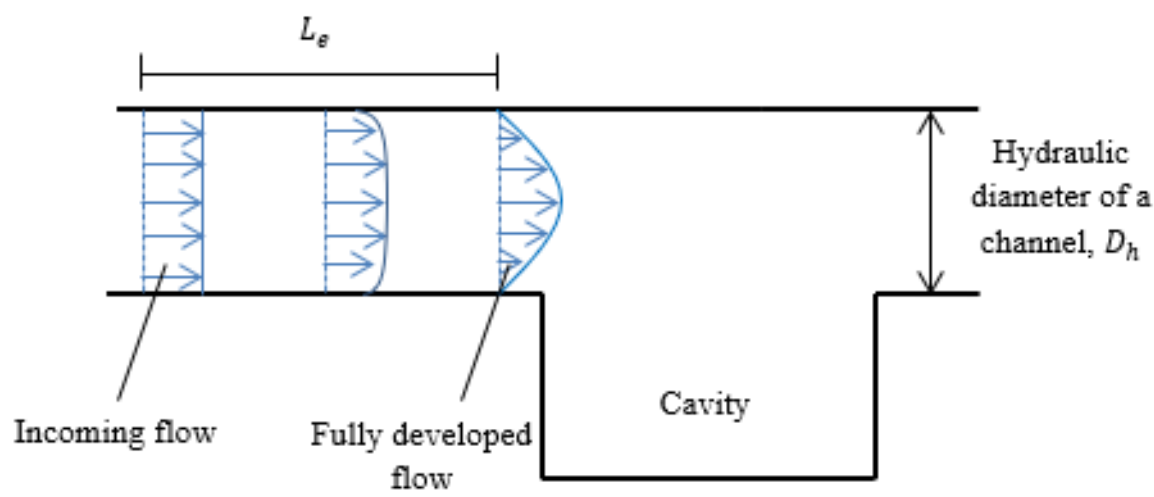

Fig. 2. The definition of entrance length $\left(L_{e}\right)$ in the channel before the cavity

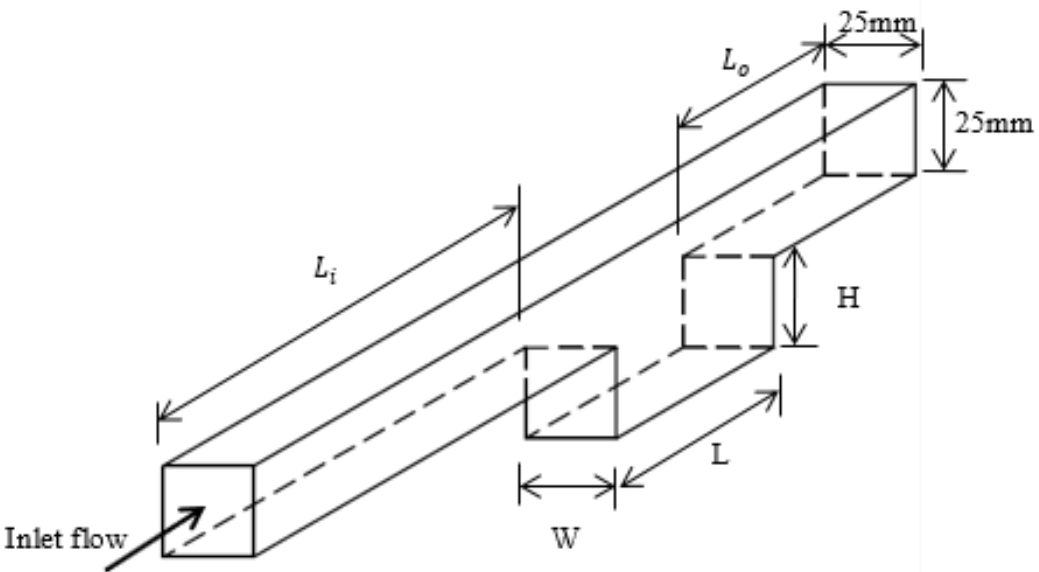

Table 2

Fig. 3. Cavity channel geometric parameters

\begin{tabular}{llllll}
\multicolumn{2}{l}{ Summary of test section dimension } & & & & \\
Aspect Ratio $(A R)$ & $L_{i}(\mathrm{~mm})$ & $L_{o}(\mathrm{~mm})$ & $L(\mathrm{~mm})$ & $W(\mathrm{~mm})$ & $H(\mathrm{~mm})$ \\
4 & 1500 & 500 & 100 & 25 & 25 \\
3 & 1525 & 500 & 75 & 25 & 25
\end{tabular}

There are two different sizes of cavities used in this experiment to represent two different aspect ratio $(A R)$ which is 3 and 4 . Other than comparison purpose to the numerical results within the research scopes, AR 3 and 4 were selected because longer cavity's length provides a clear image for visualization. Shorter length affecting the diffusion rate of the dye used for visualization, thus, produce an unclear image of flow structure. For each case, three different Reynolds number, Re have been used, 100, 400 and 1000. These values have been selected so that it can be compared with numerical results from the present method and works of literature within the scopes of the research. 
The Reynolds number, $R e$ is calculated from the incoming flow average velocity $\left(u_{\text {avg }}\right)$, the hydraulic diameter of the channel, $D_{h}$ and kinematic viscosity of the fluid, $v$. By definition,

$R e=\frac{u_{a v g} D_{h}}{v}$

By controlling the flow rate of the incoming flow, we can adjust to the desired velocity of the flow and therefore, controlling Reynolds number, Re of the flow as well. Using Eq. (3), for Reynolds number, $R e$ from 100 to 1000 , calculated range of flow velocity, $u_{\text {avg }}$ in the channel between 4.016 $\mathrm{mm} / \mathrm{s}$ and $40.160 \mathrm{~mm} / \mathrm{s}$. Determination of $u_{\text {avg }}$ for each $R e$ is important in order to obtain the value of flow rate required for the experiment. The flow rate value can be obtained by using Eq. (4).

$Q=u_{\text {avg }} A$

where $Q$ is the flow rate, $u_{\text {avg }}$ is the average flow velocity and $A$ is the area of the channel crosssection. This is crucial as the range of flow rate, $Q$ value needed for suitable pump and flowmeter scale selection. The flow rate based on the high-velocity range is between $0.151 \mathrm{l} / \mathrm{min}$ and 1.506 $I / \mathrm{min}$. The summary of flow rate for each $R e$ is shown in Table 3.

Table 3

Flow rate and flow with corresponding Reynolds number, $R e$

\begin{tabular}{lll}
\hline Reynolds number, $R e$ & Average flow velocity, $u_{\text {avg }}(\mathrm{mm} / \mathrm{s})$ & Flow rate, $Q(I / \mathrm{min})$ \\
\hline 100 & 4.0160 & 0.151 \\
400 & 16.064 & 0.602 \\
1000 & 40.160 & 1.506 \\
\hline
\end{tabular}

In the experiment, the flow rate was controlled by using a ball valve positioning upstream the pump and measured by glass flowmeter, Platon NG series range from $0.1 \mathrm{l} / \mathrm{min}-1.5 \mathrm{l} / \mathrm{min}$ with accuracy up to $1.25 \%$ full-scale deflection (FSD).

\subsection{Flow Visualization}

Three methods were used to visualize flows in experimental fluid dynamics which are surface flow visualization, particles tracer method and optical method [20-22]. In this study, the classical flow visualization technique using liquid dye as used by Mega et al., [23] was chosen. This is due to the limit of the qualitative aspect of the flow structure. Liquid dye technique can determine the moving instability of the shear layer past over the cavity. This method, however, has a drawback. It cannot produce excellent quality images for stationary vertical structure for Reynolds number, $R e$ below 100 and aspect ratio, $A R$ less than 2 for this experiment configuration.

The experiment is using Sony alpha 6000 ( $\alpha 6000)$, ILCE6000 model, 24 megapixels mirrorless digital camera with 16-55 mm zoom lens as image recorded device and positioned in front of cavity to visualize the velocity profile and flow behaviour with different Reynolds number, $R e$ especially in the cavity. The experiments for each Reynolds number were repeated three times to obtained reliable and consistent visual results. The reproducibility of flow visualizations was verified by recording various picture sequences with a different channel running, and by testing different dye ink-water mixture. Note that there is a saturation of the cavity with ink if the observation time is too long. Therefore, the dynamical flow structure in the cavity cannot be identified. 


\subsection{The Numerical Scheme}

Advection equation for hyperbolic type of equation can be solved by using Cubic Interpolated Pseudo-particle (CIP) method. This method is a semi-Lagrangian scheme which gives not an only less diffusive result but also stable outcome without any flux limiter [11]. Therefore, Yabe [24], Ishikawa et al., [11], and Yabe and Aoki [14] have proposed a general hyperbolic solver. The solver scheme needs to split the equation into two parts which are advection and non-advection phases.

Eqs. (5) and (6) show the vorticity transport and energy equation in dimensionless form. The equations consist of advection and non-advection part on the left- and right-hand side of the equations, respectively. The non-advection phase of the equations will be solved by using central finite difference, and the advection phase will be solved using two-dimensional CIP approach.

Momentum dimensionless equation

$\frac{\partial \Omega}{\partial \tau}+U \frac{\partial \Omega}{\partial X}+V \frac{\partial \Omega}{\partial Y}=\frac{1}{R e}\left(\frac{\partial^{2} \Omega}{\partial X^{2}}+\frac{\partial^{2} \Omega}{\partial Y^{2}}\right)+\frac{G r}{R e^{2}} \frac{\partial \theta}{\partial X}$

Energy dimensionless equation

$\frac{\partial \theta}{\partial \tau}+U \frac{\partial \theta}{\partial X}+V \frac{\partial \theta}{\partial Y}=\frac{1}{\operatorname{PrRe}(}\left(\frac{\partial^{2} \theta}{\partial X^{2}}+\frac{\partial^{2} \theta}{\partial Y^{2}}\right)$

where $\Omega$ is dimensionless vorticity, $X$ and $Y$ are dimensionless space in horizontal and vertical direction respectively, $U$ and $V$ are dimensionless velocity component in horizontal and vertical direction respectively, $G r$ is Grashof number, $R e$ is Reynolds number, $\theta$ is dimensionless temperature, $\tau$ is dimensionless time, and $\mathrm{Pr}$ is PrandtI number. In conjunction with the CIP method, Eq. (5) divided into advection and non-advection phases. Then, the split equation must be differentiated to determine the $\Omega$ and $\theta$ gradient. Thus, the differentiation results are described as the following equation.

The advection phase:

Momentum

$\frac{\partial \Omega}{\partial \tau}=-\left(U \frac{\partial \Omega}{\partial X}+V \frac{\partial \Omega}{\partial Y}\right)$

$\frac{\partial_{X} \Omega}{\partial \tau}=-\left(U \frac{\partial_{X} \Omega}{\partial X}+V \frac{\partial_{X} \Omega}{\partial Y}\right)$

$\frac{\partial_{Y} \Omega}{\partial \tau}=-\left(U \frac{\partial_{Y} \Omega}{\partial X}+V \frac{\partial_{Y} \Omega}{\partial Y}\right)$

Energy

$\frac{\partial \theta}{\partial \tau}=-\left(U \frac{\partial \theta}{\partial X}+V \frac{\partial \theta}{\partial Y}\right)$

$\frac{\partial_{x} \theta}{\partial \tau}=-\left(U \frac{\partial_{x} \theta}{\partial X}+V \frac{\partial_{x} \theta}{\partial Y}\right)$

$\frac{\partial_{Y} \theta}{\partial \tau}=-\left(U \frac{\partial_{Y} \theta}{\partial X}+V \frac{\partial_{Y} \theta}{\partial Y}\right)$ 
and the non-advection phase

Momentum

$\frac{\partial \Omega}{\partial \tau}=\frac{1}{R e}\left(\frac{\partial^{2} \Omega}{\partial X^{2}}+\frac{\partial^{2} \Omega}{\partial Y^{2}}\right)+\frac{G r}{R e^{2}} \frac{\partial \theta}{\partial X}$

$\frac{\partial_{X} \Omega}{\partial \tau}=\frac{1}{R e}\left(\frac{\partial^{3} \Omega}{\partial X^{3}}+\frac{\partial^{3} \Omega}{\partial X \partial Y^{2}}\right)-\partial_{X} \Omega \frac{\partial U}{\partial X}-\partial_{Y} \Omega \frac{\partial V}{\partial X}+\frac{G r}{R e^{2}} \partial_{X} \frac{\partial \theta}{\partial X}$

$\frac{\partial_{Y} \Omega}{\partial \tau}=\frac{1}{R e}\left(\frac{\partial^{3} \Omega}{\partial X^{2} \partial Y}+\frac{\partial^{3} \Omega}{\partial Y^{3}}\right)-\partial_{X} \Omega \frac{\partial U}{\partial Y}-\partial_{Y} \Omega \frac{\partial V}{\partial Y}+\frac{G r}{R e^{2}} \partial_{X} \frac{\partial \theta}{\partial Y}$

Energy

$\frac{\partial \theta}{\partial \tau}=\frac{1}{\operatorname{PrRe}}\left(\frac{\partial^{2} \theta}{\partial X^{2}}+\frac{\partial^{2} \theta}{\partial Y^{2}}\right)$

$\frac{\partial_{X} \theta}{\partial \tau}=\frac{1}{\operatorname{PrRe}}\left(\frac{\partial^{3} \theta}{\partial X^{3}}+\frac{\partial^{3} \theta}{\partial X \partial Y^{2}}\right)-\partial_{X} \theta \frac{\partial U}{\partial X}-\partial_{Y} \theta \frac{\partial V}{\partial X}$

$\frac{\partial_{Y} \theta}{\partial \tau}=\frac{1}{\operatorname{PrRe}}\left(\frac{\partial^{3} \theta}{\partial X^{2} \partial Y}+\frac{\partial^{3} \theta}{\partial Y^{3}}\right)-\partial_{X} \theta \frac{\partial U}{\partial Y}-\partial_{Y} \theta \frac{\partial V}{\partial Y}$

where $\partial_{X} \Omega=\partial \Omega / \partial \mathrm{X}$ and $\partial_{Y} \Omega=\partial \Omega / \partial \mathrm{Y}, \partial_{X} \theta=\partial \theta / \partial \mathrm{X}$ and $\partial_{Y} \theta=\partial \theta / \partial \mathrm{Y}$. The details description of the advection and non-advection phase is documented in [25] and will not be given here.

\subsection{Validation Study}

\subsubsection{Lid-driven cavity}

To simulate and validate the flow phenomenon, square cavity with wall boundary $H=L$ was introduced as shown in Figure 4. The velocity of the top lid was dimensionless where $U=u / u_{\infty}=$ 1. For the simulation, the time resolution used based on the Courant-Friedrichs-Lewy, CFL condition of Eq. (19). $\Delta \tau=0.0001$ is used in this research, which is satisfied with the CFL requirements where

$C=U \Delta \tau / \Delta X \leq C_{\max }$

where $C_{\max }=1$. Von Neumann Stability analysis procedure is used to verify the stability of finite difference schemes as applied to linear partial differential equations. Referring to the Von Neumann stability analysis, as discussed in Anderson et al., [26], the stability condition for this particular equation is approximated as follows:

$\frac{\Delta \tau}{R e}\left[\frac{1}{(\Delta X)^{2}}+\frac{1}{(\Delta Y)^{2}}\right] \leq \frac{1}{2}$ 


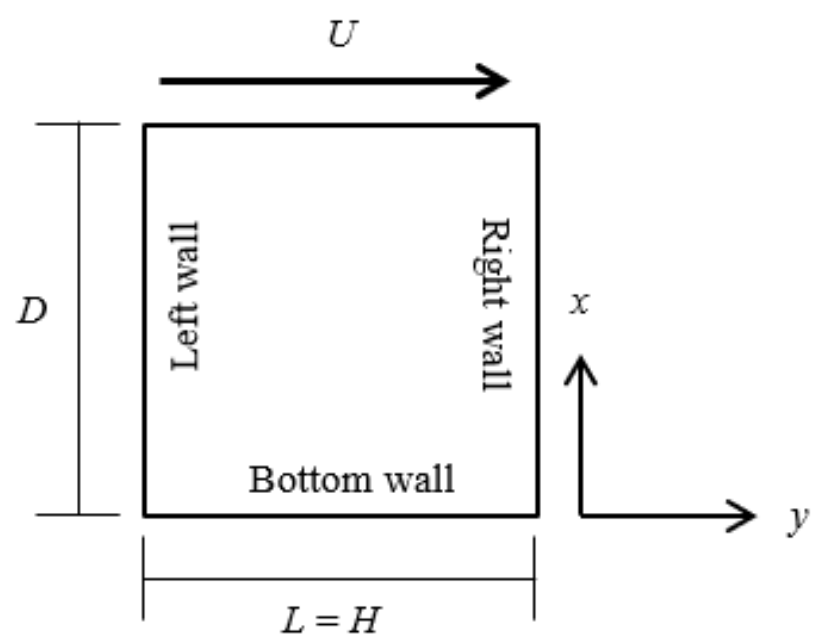

Fig. 4. Computational domain's schematic diagram and boundary conditions for validation study in a lid-driven cavity

Furthermore, the computational domain for validation work is discretized using the square element with $\Delta X=\Delta Y$. A different number of square cells depend on the number of grid points needed in the validation study. The size of the cell, $\Delta X \times \Delta Y$ is depending on the requirement from Eqs. (19) and (20) to maintain its stability and accuracy of the results. In this research, the following convergence criterion is applied to guarantee that the dependent variables converge as in Eq. (21).

$\in=\frac{\left\|\Lambda^{n+1}-\Lambda^{n}\right\|_{\infty}}{\left\|\Lambda^{n+1}\right\|_{\infty}} \leq 10^{-6}$

\subsubsection{Channel with heated cavity}

Instead of moving lid, flow inside the cavity was moved by the shear stress from the flow moving in the channel. Besides, there is mass transfer and exchange happened from the fluid in the channel to the cavity, depends on different variables of the flow and boundary of the computational domain. Work did by Stiriba et al., [27], Abdelmassih et al., [28], and Manca et al., [29] were analyzed to validate or verify the numerical method of thermal CIP in the present research.

The geometry of the channel including the square open cavity and the computational domain used in the validation study are shown as in Figure 5 with a uniform heat flux heats the bottom wall of the cavity while the other walls are adiabatic. The same configuration can be found in work by previously stated literature. However, Stiriba et al., [27], and Abdelmassih et al., [28] studied in the three-dimensional problem rather than two-dimensional as in the present study. Despite that, the required information for the validation study was provided by both works of literature. Also, Poisseulle flow enters through the left boundary, and the flow is considered to be two-dimensional, laminar and incompressible with viscous dissipation is neglected as described in Manca et al., [29]. 


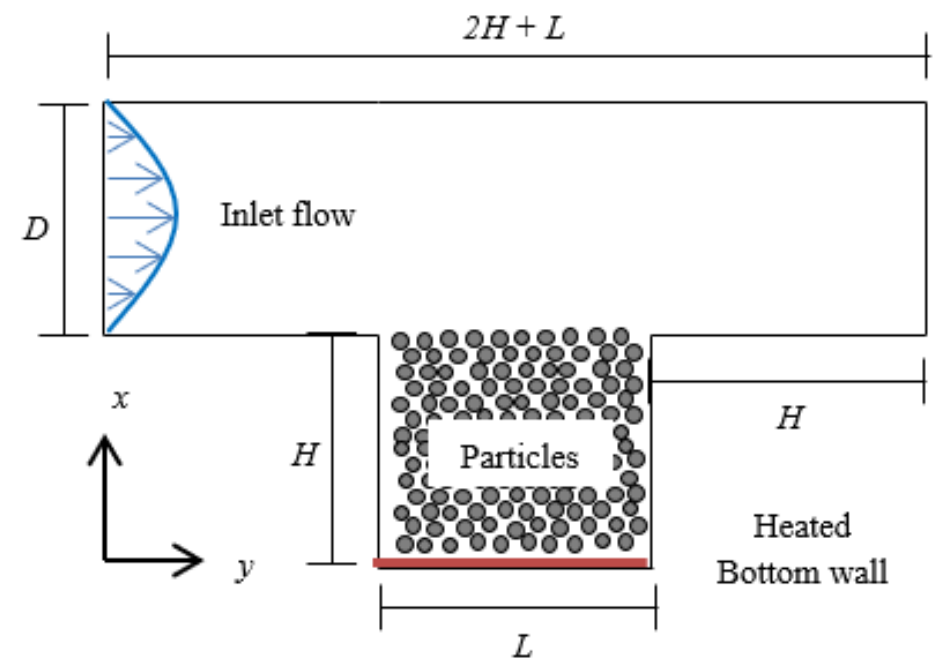

Fig. 5. Mathematical model of the channel with cavity and heat source at the bottom of the cavity for the validation study

In order to simulate and validate the flow phenomenon of the channel with a heated cavity, cavity with aspect ratio, $A R=1(L / W=1)$ and $2(L / W=2)$ was used. The computational domain for validation work was discretized using the square element with $\Delta X=\Delta Y$. For the simulation, the time resolution used based on the CFL condition of Eq. (20). $\Delta \tau=0.001$ is used in this research, which is satisfied with the $\mathrm{CFL}$ requirements. The spatial resolutions generated for the study and the parameters involved were presented as in Table 4. In this validation process, the following enforced convergence criterion is applied to guarantee that the dependent variables converge as in Eq. (21)

\section{Table 4}

Cell size $(\Delta X \times \Delta Y)$ for the cavity and flow parameters used for validation study in the channel with a heated cavity

\begin{tabular}{|l|l|l|l|l|}
\hline Cell size $(\Delta X \times \Delta Y)$ & $\operatorname{Re}$ & Gr & $A R$ & $\operatorname{Pr}$ \\
\hline $0.01 \times 0.01$ & 100 & 1000,10000 and 100000 & 1 and 2 & 0.7 \\
\hline
\end{tabular}

\section{Results}

\subsection{Validation of Numerical Results in Lid-Driven Cavity}

Figure 6 shows the comparisons of the numerical simulation results for the streamlines inside the square cavity. The results are presented in three different Re, which are 100, 400 and 1000 using the same uniform grid points $(129 \times 129)$. Figure 6 clearly illustrate that the vortex structure is affected by $\mathrm{Re}$. In the case, $\mathrm{Re}=100$, the flow in the cavity is described by one large primary vortex. As $\mathrm{Re}$ increases from 100 to 1000, the centre of primary vortex drifts off towards the centre of the cavity as a result of the effect of force inertia of the fluid.

For $R e=100$, the secondary vortices are almost invisible for displayed clearly in the streamlines. Ghia et al., [30], however, proving those secondary vortices exist in both right and left bottom corner of the cavity but in a small form. Moved to $R e=400$, as the primary vortex moved towards the centre, the secondary vortices are getting larger in shape, especially the right bottom vortex. It is significantly larger than the opposite. The strength growth of the primary vortex also increases the size and strength of the secondary vortices, directly translate into the changes of velocity of the circulating flow. The profile of the velocity of the flow will be discussed in the next section. As $R e$ is increased to 
1000 , the primary vortex is getting close to the centre of the cavity. Both secondary vortices' magnitude at the bottom corner of the cavity is getting larger due to increasing of shear from the primary vortex. Therefore, from Figure 6 , the streamlines plotted in the cavity reflected that the current results are giving a good agreement with the benchmark results.

Present uniform grid $(129 \times 129)$

Ghia et al., [31] uniform grid $(129 \times 129)$

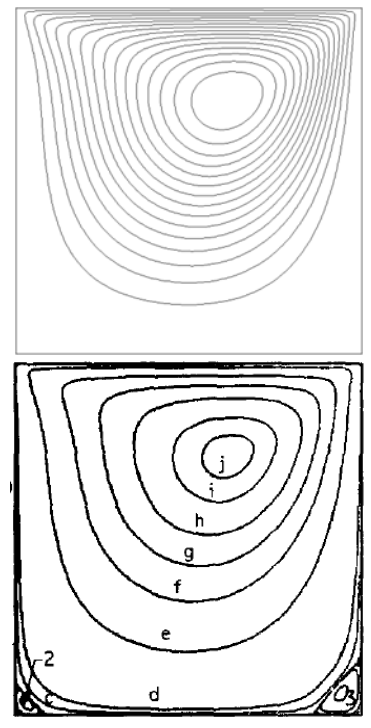

(a)

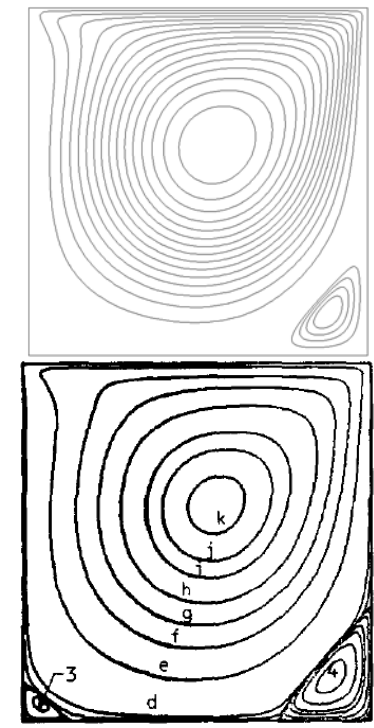

(b)

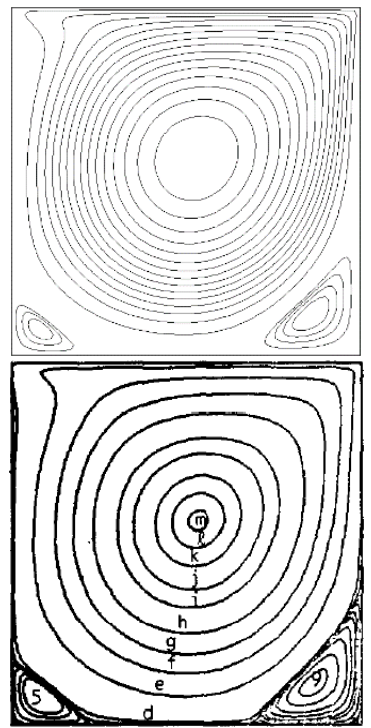

(c)

Fig. 6. Streamlines inside the square cavity based on the present numerical method and the benchmark results for (a) $R e=100$, (b) 400 and (c) 1000

\subsection{Comparative Evaluation of the Experiment and Numerical Simulation}

Figure 7 shows the streamlined profile and vortices formation inside the cavity with aspect ratio, $A R$ of 4 at steady state for $R e$ of 100, 400 and 1000 for both experiment and numerical method using CIP method. The experiment was conducted at different $R e$ for a cavity with aspect ratio, $A R=4$. By taking the cavity as a reference, the centre of primary vortex for $R e=100$ located at $X=1.37$ and $Y=$ 0.60 , slightly curved downwards to the bottom of the cavity with the separation shear wall curved downwards along the $X$-direction. This is due to the inertial forces from the incoming flow inside the channel. As $R e$ increases to 400 , the primary vortex position changes towards the forward-facing step with the separation shear wall almost linear as a result of the shear from the channel flow. At $R e=400$, the centre of primary vortex located at $X=3.36$ and $Y=0.58$ with no secondary vortex were traced. Similar formations of vortices for $R e=100$ and 400 for $A R=4$ were found in the Fang et al., [31]. For $R e=1000$, the formation of secondary vortices formed in the bottom-centre and bottomleft corner of the cavity due to increases of flow circulation inside the cavity. The higher shear from primary vortex increases the circulation of secondary vortices, thus affecting the sizes of the vortices due to the increase of inertia.

The effect of different $R e$ to the speed of flow circulation can be observed in Figure 8 . At this point, the location of the primary vortex centre in the cavity is $X=3.38$ and $Y=0.57$ while the centre of secondary vortices located at $X=0.34, Y=0.38$ and $X=2.38, Y=0.22$ respectively. 


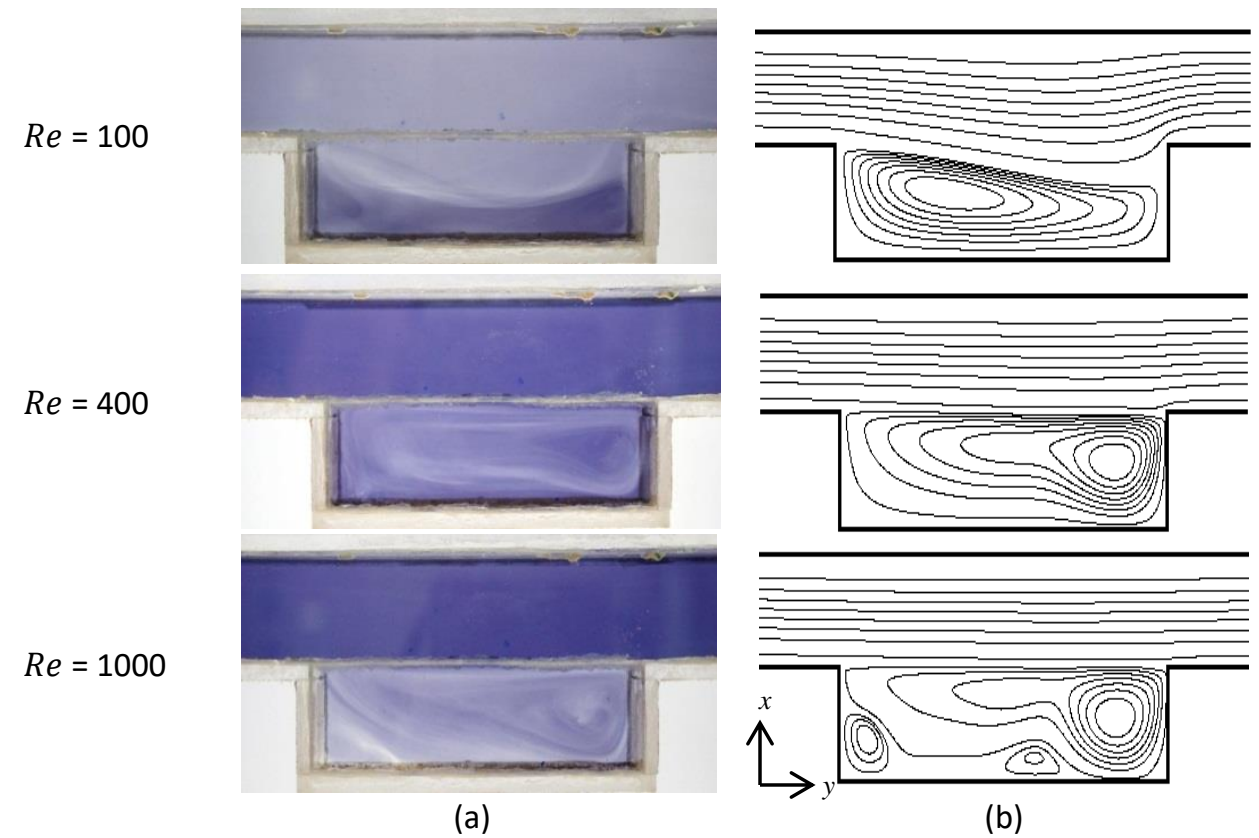

Fig. 7. Flow characteristic for aspect ratio, $A R=4$ at different Reynolds numbers, $R e(a)$ instantaneous flow visualization image obtained using dye ink and (b) numerical results using CIP method

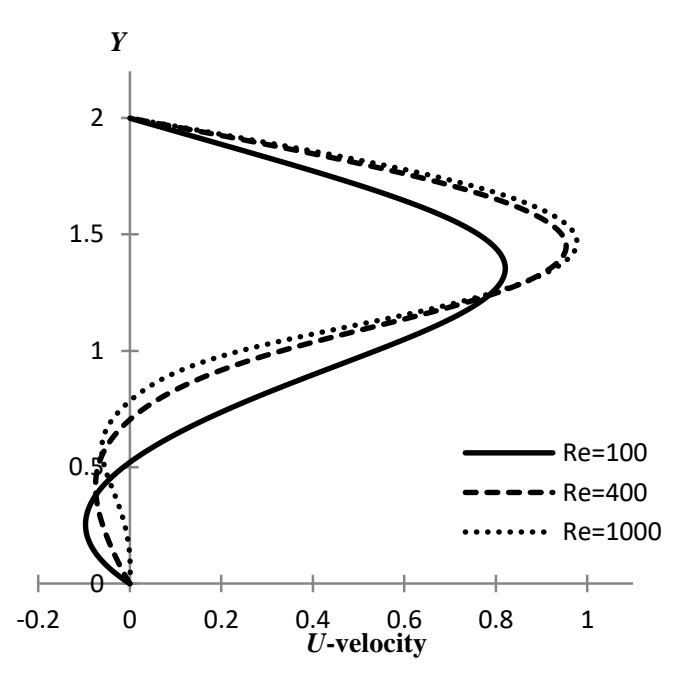

(a)

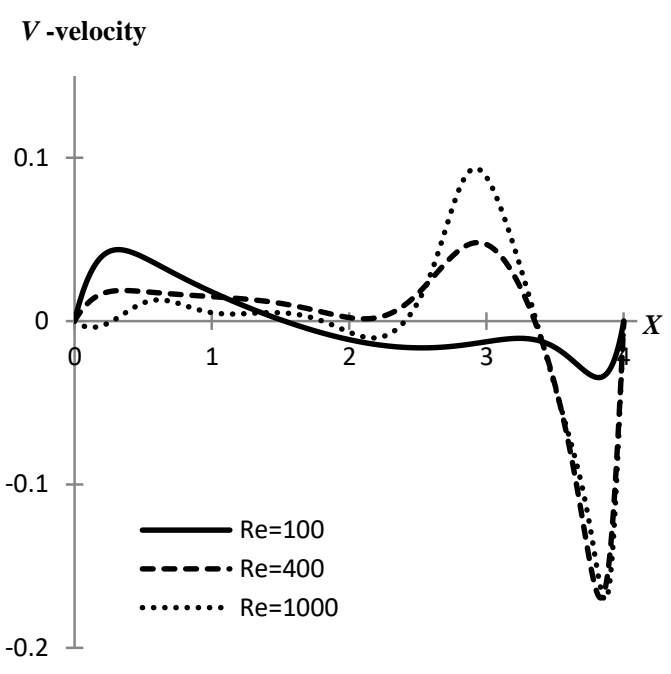

(b)

Fig. 8. Comparison of velocity components at different Reynolds number, $R e$ (a) $U$-velocity along the vertical line and (b) $V$-velocity along the horizontal line at the center of the cavity for $A R=4$

A comparison of the flow structures and vortices formation of the experimental and numerical result shows great similarities; a large primary vortex was formed in the cavity for all $R e$. The centre of the vortex moves towards the forward-facing wall for $R e=400$ when compare to $R e=100$ (see Figure 7). As $R e$ increase to 1000 , the formation of secondary vortices formed in the bottom-centre and bottom-left corner of the cavity. In the experiment results, however, secondary vortices are barely seen due to the lower intensity of the indicator dye ink in the area that may highlight the vortices formation. The differences, especially for $R e=100$, maybe due to the three-dimensional effect of the width of the cavity. The lower speed of flow circulation also made the dye ink diffused into the fluid faster. 


\subsection{Solid Particle Behaviour in a Channel Flow with Cavity}

The changes in flow's $R e$ and cavity's $A R$ significantly affect the total number of particle removal from the cavity, as shown in Figures 9 and 10. Note that when the $R e$ increasing, the percentage of particles dispersed from the cavity also increase. This effect can be observed for all $A R$. For deep cavity, $(A R=0.5)$, there are $6.5 \%$ increases in removal percentage when $R e$ is increasing from 50 to 1000. The increases are relatively small compared to other $A R$. As $R e$ increase, flow penetration into the cavity during the transient period, and the mass transfer of fluid also increases [31]. Thus, the percentage of removed particle increases.

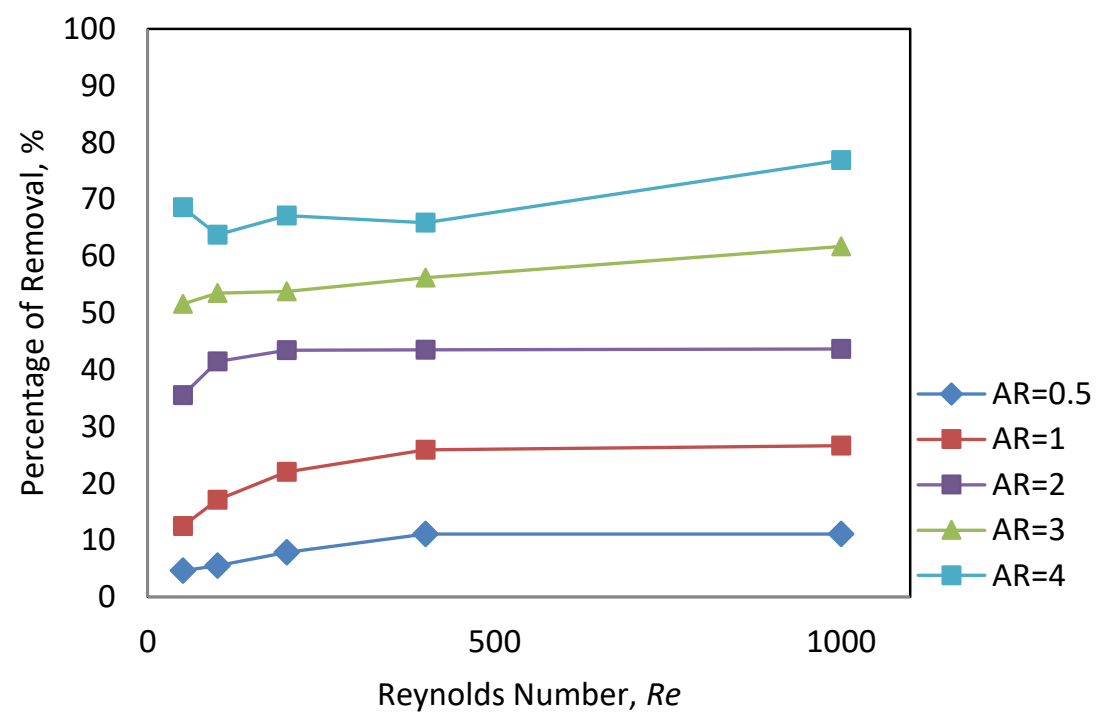

Fig. 9. Percentage of particle removal from the cavity for different Reynolds numbers, $R e$ at various aspect ratios, $A R$

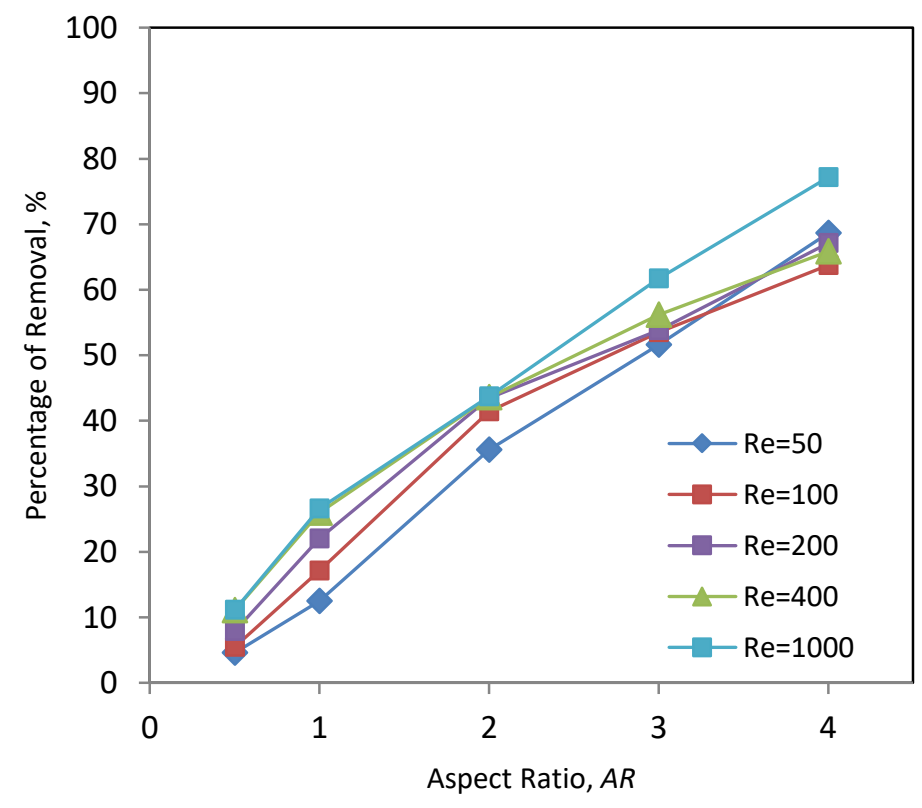

Fig. 10. Percentage of particle removal from the cavity for different aspect ratios, $A R$ at various Reynolds numbers, $R e$ 
In addition, particles dispersion behaviour was affected by the vortices formation inside the cavity. For $A R=0.5$, two vortices were seen formed as in Figure 11(a) where the primary vortex is induced by inertial forces of fluid flow of the channel while the secondary vortex is formed from the inertial forces from the primary. Therefore, as $R e$ increases, the primary vortex seems to appear skew to the right, following the direction of flow inside the channel. The changing shape and velocity of the primary vortex affecting the secondary, the formation of secondary vortex made the particles trap into the circulation of flow without enough drag to disperse out of the cavity. Furthermore, the primary vortex has a high value of kinetic energy, and the strength reduces rapidly for the secondary vortex as the cavity going deeper [32]. Therefore, in a deep cavity, the primary vortex is influential on the mass transfer of the fluid, thus, affecting the dispersion of particles out of the cavity. The changes in velocities magnitude affected by the $R e$ can be observed, as shown in Figure 12.

Closer examination on the cavity with unity $A R$, the removal percentage increases from $12.5 \%$ for $R e=50,17.125 \%(R e=100), 22 \%(R e=200), 25.9 \%(R e=400)$ to $26.625 \%$ for $R e=1000$. Higher penetration of the flow during the transient period as $R e$ increases affecting the number of particles dispersed from the cavity. A single primary vortex is formed inside the cavity, as shown in Figure 10(b) and the centre of the vortex shifted to the right as $R e$ increase. Besides, velocities magnitude of flow circulation increases as $R e$ increases as illustrated in Figure 12. As a result, particles seem to concentrate at the higher velocity region along the peripheral region of the vortex, as illustrated in Figure 11(b) due to inertia of the particles [33].

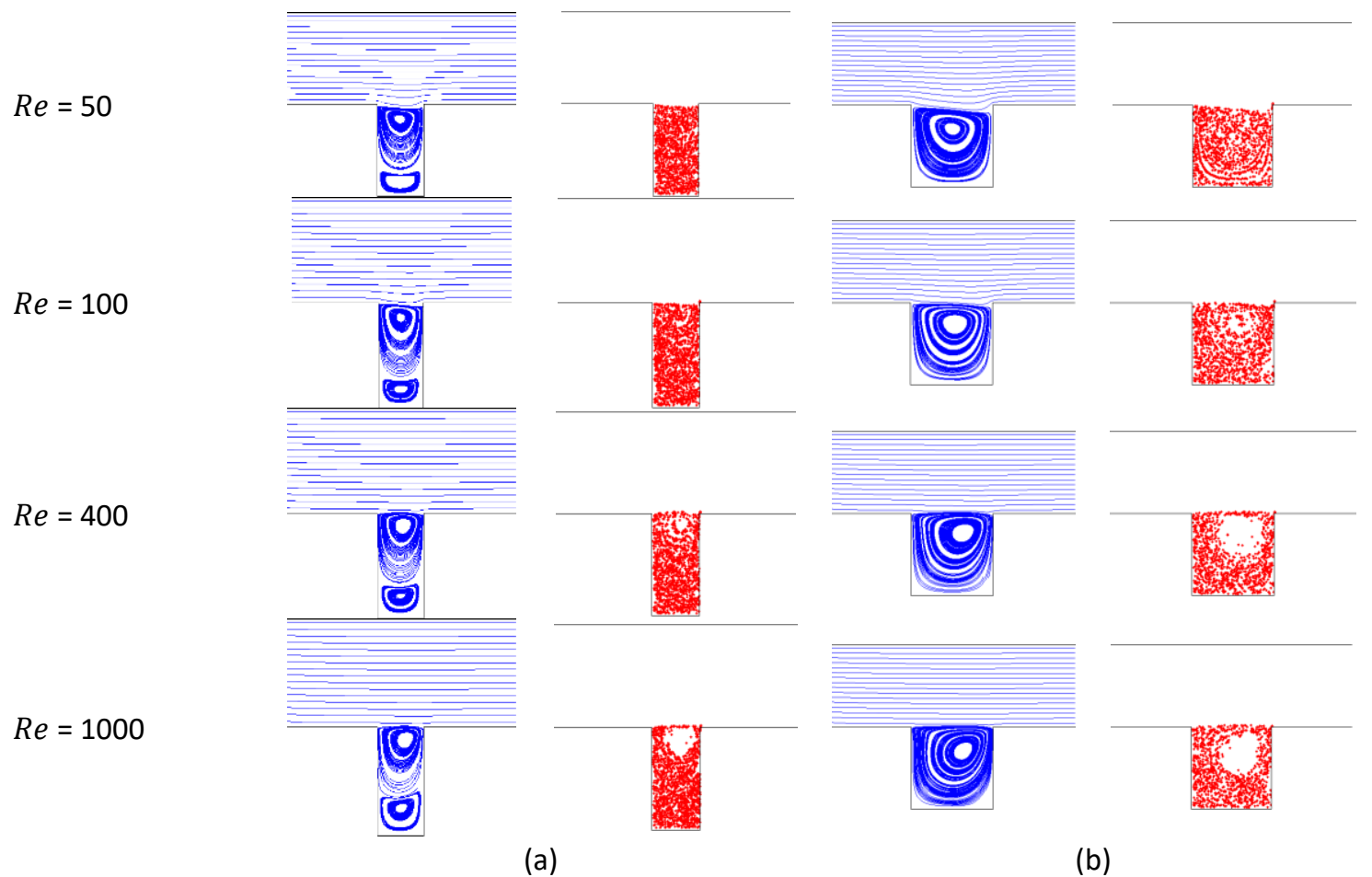

Fig. 11. Flow structure and particle distribution in the flow field of the channel with cavity with aspect ratio, $A R$ (a) 0.5 and (b) 1 at steady state at various Reynolds number, $R e$

Meanwhile, for shallow cavity, the removal percentage is increasing from $35.6 \%$ to $43.6 \%$ ( $A R=$ 2), $51.6 \%$ to $61.7 \%(A R=3)$ and $68.6 \%$ to $76.9 \%(A R=4)$ as $R e$ is increase from 50 to 1000 . Higher flow penetration during initial flow development made the shallow cavity have a higher removal percentage compared to the deep and unity cavity. By examine the removal percentage for $A R=4$, there are decreasing removal percentage $(-5 \%)$ as $R e$ increases from 50 to 100 before increases to higher $R e$. This phenomenon is opposite to other $A R$. 

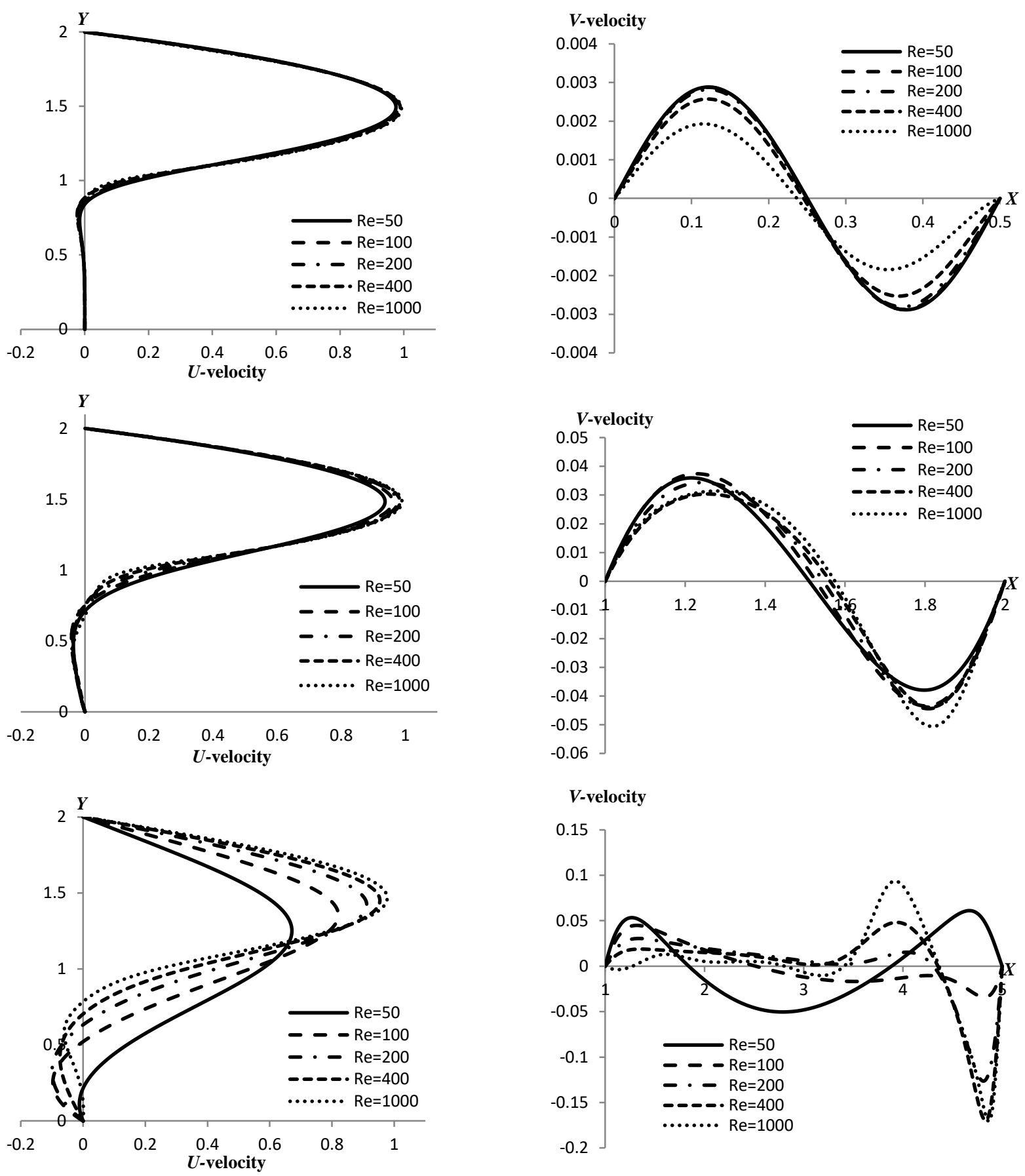

(a)

(b)

Fig. 12. Comparison of the velocity profile of aspect ratio, $A R=0.5$ (top), $A R=1$ (middle) and $A R=4$ (bottom) along the centerline of the cavity at different Reynolds number, $R e$ for (a) velocity profile of horizontal and (b) vertical component along the centerline of the cavity

For understanding the situation, a closer examination should be taken upon the flow development in the cavity for $A R=4$. As seen in Figure 13, a small vortex formed after the backwardsfacing step for $R e=50$ while large vortex was formed, and stretch to fill the cavity as $R e=100$. As a result, more particles trapped inside the circulation without being removed from the cavity compared to $R e=50$. For higher $\operatorname{Re}(200$ to 1000 ) even though the primary vortex is more extensive, most of the particles were removed during the transient period. It can be observed that the particles were 
dispersed along with the circulation at the higher velocity and vorticity region. Similar particle behaviour in vorticity region can be found from the previous study by Brandon and Aggarwal [34]. This can be observed of higher concentration of particles along the peripheral of closed circulation of the flow. Besides, the presence of two secondary vortices trapped the particles inside the vortices region as $R e=1000$.

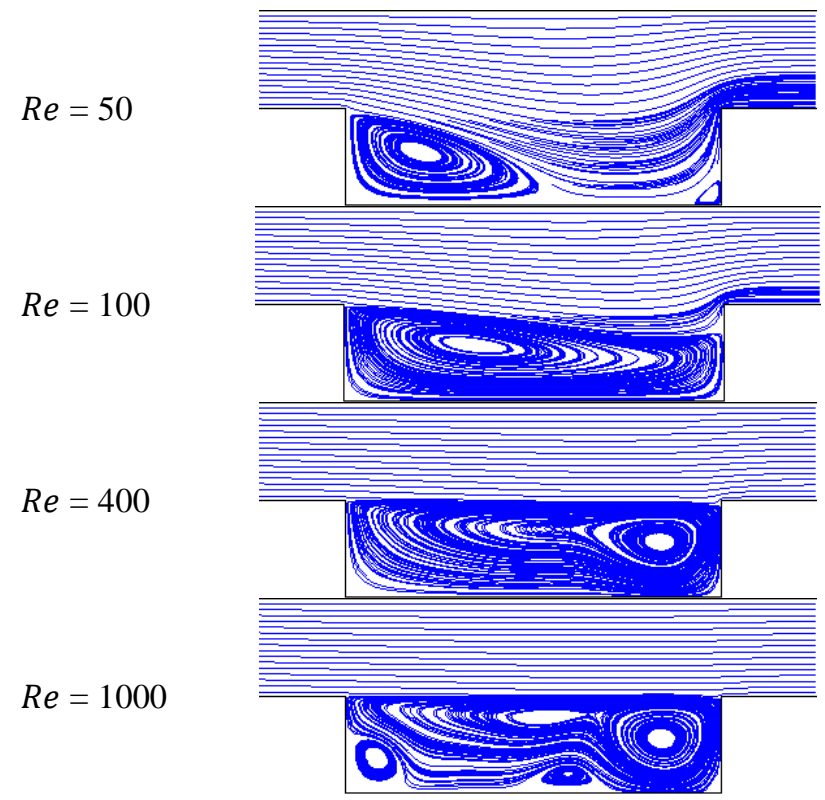

(a)

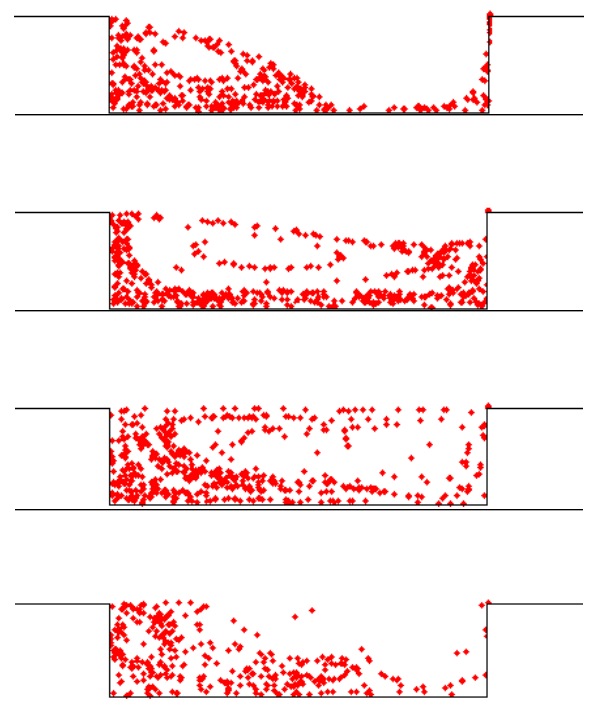

(b)

Fig. 13. Flow structure (a) and particle distribution (b) in the flow field of the channel with cavity with aspect ratio, $A R=4$ at steady state at various Reynolds number, $R e$

\section{Conclusions}

In the present study, it shows that the flow characteristic is dependent on the $A R$ of the cavity. It is good to mention that $R e$ of the flow also affects the flow and the formation of the vortices inside the cavity. For instance, for the same value of $R e$, the difference of formation of vortices structure can be observed for different $A R$. An example of these differences can be observed for Reynolds number, $R e=1000$ in the cavity with $A R=4$ where two secondary vortices are formed in a different location, where only one secondary vortex is formed in lower $A R$. The formation of vortices inside the cavity also affected the mass transfer of the fluid in the cavity.

The qualitative study of the behaviour of the particles in present results shows that the particles are dependent on the flow characteristics inside the cavity. The particles move as a result of the drag force of the particles from the fluid. From the results presented, particles tend to move along the peripheral of the vortices as those regions have higher vorticity value. The fluid flow into the cavity and formation of the vortices made a portion of the particles washed away from the cavity and the others trapped inside the circulation of the flow. These effects of the removal of particles from the cavity are most pronounced for $A R=4$ with $R e=1000$, where more flow penetration and displacement of fluid and the effect of vortices formation process inside the cavity while the particles removed least pronounced for $A R=0.5$ with $R e=50$.

\section{Acknowledgement}

This research was not funded by any grant. 


\section{References}

[1] Zhu, H. P., Z. Y. Zhou, R. Y. Yang, and A. B. Yu. "Discrete particle simulation of particulate systems: theoretical developments." Chemical Engineering Science 62, no. 13 (2007): 3378-3396.

https://doi.org/10.1016/j.ces.2006.12.089

[2] Che Sidik, Nor Azwadi, Leila Jahanshaloo, Azlin Ismail, and Alireza Fazeli. "The Use of Lattice Boltzmann Method for Particulate Flow Analysis." In Applied Mechanics and Materials, vol. 695, pp. 413-417. Trans Tech Publications Ltd, 2015. https://doi.org/10.4028/www.scientific.net/AMM.695.413

[3] Alkire, Richard C., David B. Reiser, and Robert L. Sani. "Effect of fluid flow on removal of dissolution products from small cavities." Journal of the Electrochemical Society 131, no. 12 (1984): 2795. https://doi.org/10.1149/1.2115410

[4] Yusof, Siti Nurul Akmal, Yutaka Asako, Mohammad Faghri, Lit Ken Tan, Nor Azwadi Bin Che Sidik, and Wan Mohd Arif Bin Aziz Japar. "Energy equation of swirling flow in a cylindrical container." International Communications in Heat and Mass Transfer 108 (2019): 104288. https://doi.org/10.1016/j.icheatmasstransfer.2019.104288

[5] Nobach, Holger, and Eberhard Bodenschatz. "Limitations of accuracy in PIV due to individual variations of particle image intensities." Experiments in fluids 47, no. 1 (2009): 27-38.

https://doi.org/10.1007/s00348-009-0627-4

[6] Tsorng, S. J., H. Capart, J. S. Lai, and D. L. Young. "Three-dimensional tracking of the long time trajectories of suspended particles in a lid-driven cavity flow." Experiments in Fluids 40, no. 2 (2006): 314-328.

https://doi.org/10.1007/s00348-005-0070-0

[7] Abdulkadir, M., V. Hernandez-Perez, S. Lo, I. S. Lowndes, and B. J. Azzopardi. "Comparison of experimental and computational fluid dynamics (CFD) studies of slug flow in a vertical 90 bend." The Journal of Computational Multiphase Flows 5, no. 4 (2013): 265-281.

https://doi.org/10.1260/1757-482X.5.4.265

[8] Chu, K. W., and A. B. Yu. "Numerical simulation of complex particle-fluid flows." Powder Technology 179, no. 3 (2008): 104-114. https://doi.org/10.1016/i.powtec.2007.06.017

[9] Elghobashi, Said. "On predicting particle-laden turbulent flows." Applied scientific research 52, no. 4 (1994): 309329.

https://doi.org/10.1007/BF00936835

[10] Yabe, Takashi, and Eiji Takei. "A new higher-order Godunov method for general hyperbolic equations." Journal of the Physical Society of Japan 57, no. 8 (1988): 2598-2601.

https://doi.org/10.1143/JPSJ.57.2598

[11] Ishikawa, T., P. Y. Wang, T. Aoki, Y. Kadota, and F. Ikeda. "A universal solver for hyperbolicequations by cubicpolynomial interpolation. 2. 2-dimensional and 3-dimensional solvers." Comput. Phys. Commun 66 (1991): 233242. https://doi.org/10.1016/0010-4655(91)90072-S

[12] Takewaki, H., A. Nishigushi, and T. Yabe. "Cubic interpolated pseudo-particle method (CIP) for solving hyperbolic type equation." National Institute for Fusion Science. NII-Electronic Library Services (1985). https://doi.org/10.1016/0021-9991(85)90085-3

[13] Takewaki, Hideaki, and Takashi Yabe. "The cubic-interpolated pseudo particle (CIP) method: application to nonlinear and multi-dimensional hyperbolic equations." Journal of Computational Physics 70, no. 2 (1987): 355372. https://doi.org/10.1016/0021-9991(87)90187-2

[14] Yabe, Takashi, and T. Aoki. "A universal solver for hyperbolic equations by cubic-polynomial interpolation I. Onedimensional solver." Computer physics communications 66, no. 2-3 (1991): 219-232. https://doi.org/10.1016/0010-4655(91)90071-R

[15] Ishikawa, T., P. Y. Wang, T. Aoki, Y. Kadota, and F. Ikeda. "A universal solver for hyperbolicequations by cubicpolynomial interpolation. 2. 2-dimensional and 3-dimensional solvers." Comput. Phys. Commun 66 (1991): 233242. https://doi.org/10.1016/0010-4655(91)90072-S

[16] Shi, YuFeng, Biao Xu, and Yan Guo. "A compact-type CIP method for general Korteweg-de Vries equation." In Abstract and Applied Analysis, vol. 2014. Hindawi, 2014.

https://doi.org/10.1155/2014/640194 
[17] Ida, Masato. "An improved unified solver for compressible and incompressible fluids involving free surfaces. Part I. Convection." Computer Physics Communications 132, no. 1-2 (2000): 44-65.

https://doi.org/10.1016/S0010-4655(00)00136-3

[18] Azwadi, Nor, A. S. Ahmad Sofianuddin, and K. Y. Ahmat Rajab. "Transient Removal of Contaminants in Cavity of Mixed Convection in a Channel by Constrained Interpolated Profile Method." Applied Mechanics \& Materials 554 (2014). https://doi.org/10.4028/www.scientific.net/AMM.554.312

[19] Sheldareh, Ali Akbari, Arman Safdari, and Nor Azwadi Che Sidik. "Numerical prediction of heat transfer from localized heating in enclosure using CIP method." In Applied Mechanics and Materials, vol. 315, pp. 512-516. Trans Tech Publications Ltd, 2013. https://doi.org/10.4028/www.scientific.net/AMM.315.512

[20] Molki, Arman, Lyes Khezzar, and Afshin Goharzadeh. "Measurement of fluid velocity development in laminar pipe flow using laser Doppler velocimetry." European Journal of Physics 34, no. 5 (2013): 1127. https://doi.org/10.1088/0143-0807/34/5/1127

[21] Smits, A. J., and Lim, T. T. Flow visualization: Techniques and examples. Imperial College Press. ISBN 1-86094-193$1,2000$. https://doi.org/10.1142/p167

[22] Settles, Gary S. Schlieren and shadowgraph techniques: visualizing phenomena in transparent media. Springer Science \& Business Media, 2012.

[23] Samimy, Mohammad, Kenneth S. Breuer, L. Gary Leal, and Paul H. Steen, eds. "A gallery of fluid motion." (2004): 1262-1263. https://doi.org/10.1119/1.1778399

[24] Mega, Eduardo F., Vinicius S. Morais, Edson DR Vieira, and Sérgio S. Mansur. "EXPERIMENTAL VISUALIZATION OF THE FLOW INSIDE OPEN CAVITIES." (2007).

[25] A. Sahak, Ahmad Sofianuddin. "Cubic Interpolation Pseudo-Particle Navier-Stokes Formulation Method for ParticleFluid Interaction in Cavity." PhD diss., Universiti Teknologi Malaysia, 2020.

[26] Anderson, D. A., Tannehill, J. C., and Pletcher, R. H. Computational Fluid Mechanics and Heat Transfer. Washington, Hemisphere Pub. Corp, 1984.

[27] Stiriba, Y. F. J. A., J. A. Ferré, and F. X. Grau. "Heat transfer and fluid flow characteristics of laminar flow past an open cavity with heating from below." International Communications in Heat and Mass Transfer 43 (2013): 8-15. https://doi.org/10.1016/j.icheatmasstransfer.2013.01.010

[28] Abdelmassih, Gorg, Anton Vernet, and Jordi Pallares. "Steady and unsteady mixed convection flow in a cubical open cavity with the bottom wall heated." International Journal of Heat and Mass Transfer 101 (2016): 682-691. https://doi.org/10.1016/j.ijheatmasstransfer.2016.05.074

[29] Manca, Oronzio, Sergio Nardini, Khalil Khanafer, and Kambiz Vafai. "Effect of heated wall position on mixed convection in a channel with an open cavity." Numerical Heat Transfer: Part A: Applications 43, no. 3 (2003): 259282. https://doi.org/10.1080/10407780307310

[30] Ghia, U. K. N. G., Kirti N. Ghia, and C. T. Shin. "High-Re solutions for incompressible flow using the Navier-Stokes equations and a multigrid method." Journal of computational physics 48, no. 3 (1982): 387-411. https://doi.org/10.1016/0021-9991(82)90058-4

[31] Fang, L. C., D. Nicolaou, and J. W. Cleaver. "Transient removal of a contaminated fluid from a cavity." International journal of heat and fluid flow 20, no. 6 (1999): 605-613. https://doi.org/10.1016/S0142-727X(99)00050-8

[32] Chang, Ho Nam, Hwa Won Ryu, Deug Ho Park, Yong Seok Park, and Joong Kon Park. "Effect of external laminar channel flow on mass transfer in a cavity." International journal of heat and mass transfer 30, no. 10 (1987): 2137 2149. https://doi.org/10.1016/0017-9310(87)90092-5

[33] Safdari, Arman, and Kyung Chun Kim. "Lattice Boltzmann simulation of solid particles behavior in a threedimensional lid-driven cavity flow." Computers \& Mathematics with Applications 68, no. 5 (2014): 606-621. https://doi.org/10.1016/i.camwa.2014.07.004

[34] Brandon, Daniel J., and S. K. Aggarwal. "A numerical investigation of particle deposition on a square cylinder placed in a channel flow." Aerosol Science \& Technology 34, no. 4 (2001): 340-352. https://doi.org/10.1080/02786820121279 